\title{
Diseño multiobjetivo de un sistema híbrido eólico-solar con baterías para zonas no interconectadas
}

\section{Multiobjective design for a hybrid solar-wind-battery system operating in non-interconnected areas}

\author{
Alejandro Castillo Ramírez \\ Ingeniero de Materiales. Estudiante de Maestría en Ingeniería de la Universidad \\ de Antioquia. Medellín, Colombia. Contacto: alcara52@hotmail.com
}

\section{Fernando Villada Duque}

Ingeniero Electricista. Docente de la Facultad de Ingeniería de la Universidad de Antioquia. Medellín, Colombia. Contacto: fvillada@udea.edu.co

\section{Jaime Alejandro Valencia Velásquez}

Ingeniero Electricista. Docente de la Facultad de Ingeniería de la Universidad de Antioquia. Medellín, Colombia. Contacto: jvalenci@udea.edu.co

Fecha de recepción: 29 de agosto de 2012

Clasificación del artículo: investigación

Fecha de aceptación: 21 de mayo de 2013

Financiamiento: Universidad de Antioquia

Palabras clave: baterías, costos nivelados de generación, probabilidad de pérdida de suministro, sistemas híbridos de energía.

Keywords: batteries, levelized cost of energy, loss of power supply probability, hybrid power systems.

\section{RESUMEN}

La energía solar y la energía eólica son dos de las principales fuentes naturales de energía intermitente en el mundo. La combinación de estas en sistemas híbridos ha demostrado ser complementaria para maximizar su confiabilidad. A continuación presentamos una metodología cronológica basada en las técnicas de Hongxing y Diaf para configurar de manera óptima un Sistema Híbrido Eólico-Solar con baterías para almacenamiento de energía (Hybrid Photovoltaic Wind System- HPWS). Asimismo, se tiene como propósito promocionar el concepto de sistemas híbridos desde una perspectiva teórica. El modelo puede ser usado para optimizar un sistema minimizando la Probabilidad de Pérdida de Suministro de la Energía (Loss Power Supply Probability- LPSP), así como los Costos 
Anualizados de lo que conlleva su gestión (Annualized Cost of System- ACS). La metodología propuesta fue usada para diseñar un HPWS aislado con el fin de proveer la demanda de energía de 49 usuarios de la ranchería Ishiruwo localizada en el departamento de La Guajira (Colombia). Los resultados muestran que existe un comportamiento inversamente proporcional entre los 2 objetivos evaluados. La solución elegida revela un bajo costo en compañía de una alta confiabilidad, sugiriendo que es mejor utilizar un sistema totalmente fotovoltaico, pues el costo de las turbinas eólicas resultó ser oneroso. Finalmente, es evidente que el costo de los sistemas aislados ha empezado a cobrar importancia porque es competitivo frente a otros tipos de generación como el diésel, pese a que más del $50 \%$ de su valor proceda de baterías $\mathrm{y}$ otros componentes.

\section{ABSTRACT}

Solar- and wind power are the two major natural sources of intermittent power in the world. The combination of these sources has proved to be a suitable combination to maximize system reli-

\section{INTRODUCCIÓN}

Algunas de las Tecnologías de Energía Renovables (Renewable Energy Technologies [RET]) como la energía solar y la energía eólica son intermitentes puesto que el recurso no está disponible a todo momento [1]. En efecto, este tipo de tecnologías no pueden seguir una curva de potencia estable. No obstante, la combinación de aquellas en sistemas híbridos ha demostrado ser complementaria para amortiguar este inconveniente y vislumbrar su posicionamiento en nuevos mercados energéticos.

En la actualidad, básicamente existen 2 formas de modelar estos sistemas. La primera correspon- ability. A chronological methodology based on Hongxing and Diaf techniques for optimal sizing of a Photovoltaic Wind Hybrid System (HPWS) (using batteries for energy storage) is presented. Accordingly, the purpose of this work is to help to promote the hybrid power system concept through a fully theoretical point of view. The model can be used to optimize the system configuration, minimizing Loss of Power Supply Probability (LPSP) and Annualized Cost of the System (ACS). The proposed methodology was used to design an isolated HPWS that meets the energy demand of 49 users at Ishiruwo settlement (located in the municipality of Uribia, State of La Guajira - Colombia). Results show that there is an inversely proportional behavior between the two objectives under evaluation. Likewise, the chosen solution with low cost and high reliability suggests that it is better to use a fully Photovoltaic System (PV), since wind-turbine cost is very high. Finally, it is clear that the cost of isolated systems has begun to gain importance due to its competitiveness when compared to other types of generation such as diesel, although more than $50 \%$ of its value lies in batteries and other components.

de a una aproximación basada en simulaciones cronológicas que usualmente requiere grandes esfuerzos computacionales, así como de la disponibilidad de datos en ciertos periodos de tiempo [2] La segunda, en cambio, utiliza una serie de técnicas probabilísticas que incorporan la naturaleza cambiante del recurso y la carga sin necesidad de datos de series de tiempo [3].

Considerando que actualmente en Colombia una gran mayoría de localidades no cuenta con suficiente información meteorológica para un estudio probabilístico, el método cronológico se propone como alternativa. Generalmente, al usar este procedimiento se minimiza el Costo Anualizado del Sistema (Annualized Cost of System [ACS]) 
así como la Probabilidad de una Pérdida de Suministro de Energía (Loss of Power Supply Probability [LPSP]) [2]. Numerosas configuraciones de capacidad para diseñar el sistema híbrido son obtenidas teniendo en cuenta diversas variables de decisión, entre las que se encuentran el número de paneles fotovoltaicos $\left(N_{p v}\right)$, el número de turbinas eólicas $\left(N_{w t}\right)$, el número de bancos de baterías $\left(N_{b a t}\right)$ y la altura de las turbinas eólicas $\left(N_{w t}\right)$.

En este documento se presenta el diseño óptimo de un sistema híbrido eólico-solar con baterías minimizando el ACS y el LPSP. El objetivo es suministrar la energía necesaria para 49 usuarios de la ranchería Ishiruwo localizada en el municipio de Uribia, departamento de La Guajira (Colombia). El escrito presenta 4 secciones posteriores, a saber: la sección del modelo, en la cual se presenta la metodología desarrollada; la sección de optimización, en la cual se describe la técnica utilizada; la sección del caso de estudio, donde se plantea un ejemplo práctico para utilizar la metodología; y finalmente la sección de conclusiones, que destaca los principales resultados, las implicaciones más relevantes y las limitaciones más importantes para desarrollar este tipo de proyectos.

\section{METODOLOGÍA}

Un HPWS con baterías básicamente está conformado por el arreglo fotovoltaico, las turbinas eólicas, el banco de baterías, los inversores, los controladores y otros componentes como los cables. Para su correcto dimensionamiento, el sistema debe evaluarse técnicamente y luego económicamente.

\subsection{Evaluación técnica}

Diversos tipos de modelos técnicos han sido desarrollados para modelar un HPWS. En esta sección se detalla el modelo matemático seguido para simular cada uno de los componentes que conforman dicho sistema. Por consiguiente, es preciso dividir esta sección en 4 subsecciones: en la primera, el modelamiento del arreglo fotovoltaico; en la segunda, el modelamiento de las turbinas eólicas; en la tercera, el modelo de las baterías, y en la cuarta el modelo de la confiabilidad del sistema.

\subsubsection{Modelo del arreglo fotovoltaico}

Para estimar la salida de potencia de los módulos fotovoltaicos (PV) se utilizó el modelo de Diaf et ál. [4],cuya expresión la podemos observar en la ecuación 1:

$$
P_{p v}=\eta_{g} \cdot N_{p v} \cdot A_{m} \cdot G_{t}
$$

Donde $\eta_{g}$ es la eficiencia instantánea del arreglo $\mathrm{PV}, A_{m}$ es el área de un módulo fotovoltaico $\left(\mathrm{m}^{2}\right)$, $G_{t}$ es la irradiancia incidente sobre un plano inclinado $\left(\mathrm{W} / \mathrm{m}^{2}\right)$ y $N_{p v}$ es el número de módulos.

\subsubsection{Modelo de las turbinas eólicas}

Para estimar la salida de potencia de las turbinas eólicas (WT) se utilizó el modelo de Hongxing et ál. [2], cuya expresión la podemos observar en la ecuación 2:

$$
P_{w}=\left\{\begin{array}{l}
P_{n} \cdot\left(V-V_{a}\right)\left(V_{n}-V_{a}\right) \rightarrow\left(V_{a} \leq V<V_{n}\right) \\
P_{n} \rightarrow\left(V_{n} \leq V<V_{f}\right) \\
0 \rightarrow\left(V<V_{a} \ldots V>V_{f}\right)
\end{array}\right.
$$

Donde $V_{a}$ es la velocidad de arranque de la turbina eólica, $V_{n}$ la velocidad nominal de la turbina eólica, $V_{f}$ la velocidad de frenado de la turbina eólica y $P_{n}$ es la potencia generada cuando la velocidad del viento (V) varía desde $V_{n}$ hasta $V_{f}$.

\subsubsection{Modelo del banco de baterías}

A cualquier hora la capacidad de la batería está relacionada con la capacidad y la energía 
producida en la hora previa. En consecuencia, para describir este comportamiento se utilizó el modelo de Bin et ál. [5], cuya expresión se muestra en la ecuación (3):

$$
C_{b a t}(t)=C_{b a t}(t-1)(1-\sigma)+\left(E_{p v}(t)+E_{w t}(t)-\left(\frac{E_{l}(t)}{\eta_{i v v}}\right)\right) \cdot \eta_{b a t}
$$

Donde $C_{b a t}(t)$ y $C_{b a t}(t-1)$ son las capacidades del banco de baterías en la hora t y $\eta_{b a t}$, es la eficiencia de la batería (durante los procesos de descarga es igual a 1, y durante los procesos de carga varía entre 0,65 a 0,85 ), es la velocidad de autodescarga del banco de baterías, $E_{p v}(t)$ y $E_{w t}(t)$ es la generación respectiva de los sistemas $\mathrm{PV}$ y WT, $E_{l}(t)$ es la carga o energía demandada a la hora t y $\eta_{i n v}$ es la eficiencia del inversor. A cualquier hora, la capacidad almacenada de la batería debe estar entre $C_{\text {batmin }} \leq C_{\text {bat }} \leq C_{\text {batmax }}$, donde $C_{\text {batmin }}$ y $C_{\text {batmax }}$ son las capacidades máxima y mínima permitidas.

\subsubsection{Requerimiento de Probabilidad de Pérdida de Suministro de la Energía (LPSP)}

Debido a la intermitencia de la radiación solar y las características del viento en cualquier lugar, el análisis de confiabilidad ha sido considerado como un factor clave para configurar un HPWS. Un sistema de generación de potencia confiable supone que el sistema tiene suficiente potencia para alimentar la demanda de carga durante un cierto periodo, o en otras palabras, que tiene una Probabilidad de Pérdida de Suministro nula (Loss of Power Supply Probability [LPSP]). Por esta razón, se buscará siempre diseñar el HPWS con una alta confiabilidad. Para modelar esto, se modificó la expresión de Hongxing et ál. [2] para simplificar los cálculos de la simulación cronológica. La ecuación (4) bosqueja dicha expresión:

$$
L P S P=\frac{\sum_{t=0}^{T} \operatorname{Veces}\left(P_{\text {disponible }}(t)<P_{\text {necesaria }}(t)\right.}{N}
$$

Donde $N$ es el número de intervalos de tiempo analizados, y $P_{\text {disponible }}$ es la potencia suministrada a la carga menos la potencia necesaria. Cabe resaltar que, en este caso, no fue necesario utilizar el área bajo la curva debido a que el objeto último de la expresión anterior consiste en determinar la probabilidad de ocurrencia del fenómeno LPSP.

\subsection{Evaluación económica}

Para cuantificar los costos del sistema se utiliza la metodología de Costos Anualizados (Annualized Costs of System [ACS]) desarrollada por Hongxing et ál. [2]. Posteriormente, se calculan los Costos Nivelados de Generación de Electricidad (Levelized Cost of Electricity [LCOE]) usando la metodología de la Agencia de Energía Nuclear (Nuclear Energy Agency [NEA]) [6] - [8], y finalmente se procede a calcular el Costo Presente Neto (Net Present Cost [NPC]) utilizando la metodología de evaluación del valor presente.

\subsubsection{Requerimiento de Costos Anualizados del Sistema (ACS)}

Hongxing et ál. [2] desarrollaron una de las metodologías más utilizadas para el análisis de costos. En el nuevo modelo, se utilizaron 3 factores: 1) el costo anual e inicial de cada uno de los componentes $\left(C_{\text {acap }}\right),(2)$ el costo anual de componentes a remplazar $\left(C_{\text {arep }}\right)$, y (3) el costo anual por mantenimiento $\left(C_{\text {amain }}\right)$ (ver ecuación (5).

$$
\begin{gathered}
A C S=C_{\text {acap }}(P V+\text { Wind }+ \text { Bat }+ \text { Tower }+ \text { Others })+1 \\
\text { Bat })+C_{\text {amain }}(P V+\text { Wind }+ \text { Bat }+ \text { Tower }+ \text { Others })
\end{gathered}
$$

\subsubsection{Requerimiento de Costos Nivelados de Generación Eléctrica (LCOE)}

Los LCOE son una herramienta muy útil para comparar los costos unitarios de las diferentes tecnologías en vida económica. En otras pala- 
bras, son los costos que le corresponden a un inversionista asumiendo la certeza de los costos de producción y la estabilidad de los precios de la electricidad. La fórmula para determinar los LCOE es mostrada en la ecuación (6):

$$
L C O E=\frac{\sum\left(I_{t}+O \& M_{t}+F_{t}+C_{t}+D_{t}\right) \cdot(1+r)^{-t}}{\sum E_{t} \cdot(1+r)^{-t}}
$$

Donde $I_{t}$ es la inversión inicial del proyecto, $O M_{t}$ es el mantenimiento proyectado del HPWS, $F_{t}$ es el costo del combustible, $C_{t}$ es el costo por contaminar en dólares por tonelada de $\mathrm{CO}_{2}$ y $D_{t}$ es el costo del desmantelamiento al final de la vida útil del proyecto. $(1+r)^{-t}$ es el factor financiero para llevar costos futuros a un valor presente.

\subsubsection{Requerimiento de Costo Presente Neto (NPC)}

El Costo Presente Neto (Net Present Cost [NPC]) es una de las metodologías más utilizadas para analizar el costo de un proyecto al inicio de su inversión. La fórmula para determinar su valor se muestra en la ecuación (7):

$$
N P C=\frac{\left.A C S \cdot(1+r)^{t}-1\right)}{r \cdot(1+r)^{t}}
$$

\section{OPTIMIZACIÓN CON ALGORITMOS GENÉTICOS}

La combinación óptima de un HPWS puede determinarse usando el mejor compromiso entre los dos objetivos considerados: la confiabilidad del sistema y los costos anuales del sistema (LPSP y ACS). Para optimizar ambas funciones se utilizó la técnica de optimización con Algoritmos Genéticos (AG). Esto, debido a la complejidad del sistema (4 variables de decisión). Particularmente, un AG es una técnica de optimización moderna programada para simular el principio de evolución natural usando 3 operadores (selec- ción, cruce y mutación). Comparada con los métodos tradicionales, una de sus ventajas consiste en su capacidad para encontrar soluciones óptimas globales, específicamente en problemas con más de un objetivo (multiobjetivo). El concepto más aceptado y generalizado de óptimo dentro del marco de los problemas multiobjetivo es el propuesto por Pareto, el cual permite descartar fácilmente numerosas configuraciones al dejar la decisión reducida a la denominada frontera de Pareto. Para optimizar ambos objetivos (LPSP y ACS), se utilizó la herramienta de optimización multiobjetivo usando el algoritmo genético por defecto del toolbox de Matlab [9]. Cabe anotar que la herramienta empleada utiliza un algoritmo genético de Clasificación No Dominada II (Non-Dominated Sorting in Genetic Algorithms [NSGA II]) [10].

\section{EXPERIMENTACIÓN Y RESULTADOS}

Para evaluar el desempeño del modelo técnicoeconómico se simuló un HPWS en un lugar promisorio de Colombia. El lugar elegido fue la Granja Paici (11 $35^{\circ} \mathrm{N} 72^{\circ}$ 19'W - Punto Negro) [11], ubicada en la ranchería Ishiruwo a pocos kilómetros al sur de Uribia en el departamento de La Guajira (figura 1), debido básicamente a que es una zona con excelente potencial de recursos eólico-solares y a su vez se caracteriza por no tener cobertura total del suministro de energía eléctrica, en tanto el $94 \%$ de las viviendas no tiene conexión [12].

Debido a los limitados recursos económicos para realizar este proyecto, no fue posible adquirir los datos climatológicos horarios de un año, no obstante, se logró comprar aquellos relativos a un mes, particularmente los del periodo con menor recurso energético. Así pues, los datos climatológicos de temperatura horaria, velocidad del viento horaria y radiación horaria para el periodo de octubre de 2008 en la estación Granja 


\section{investigación}

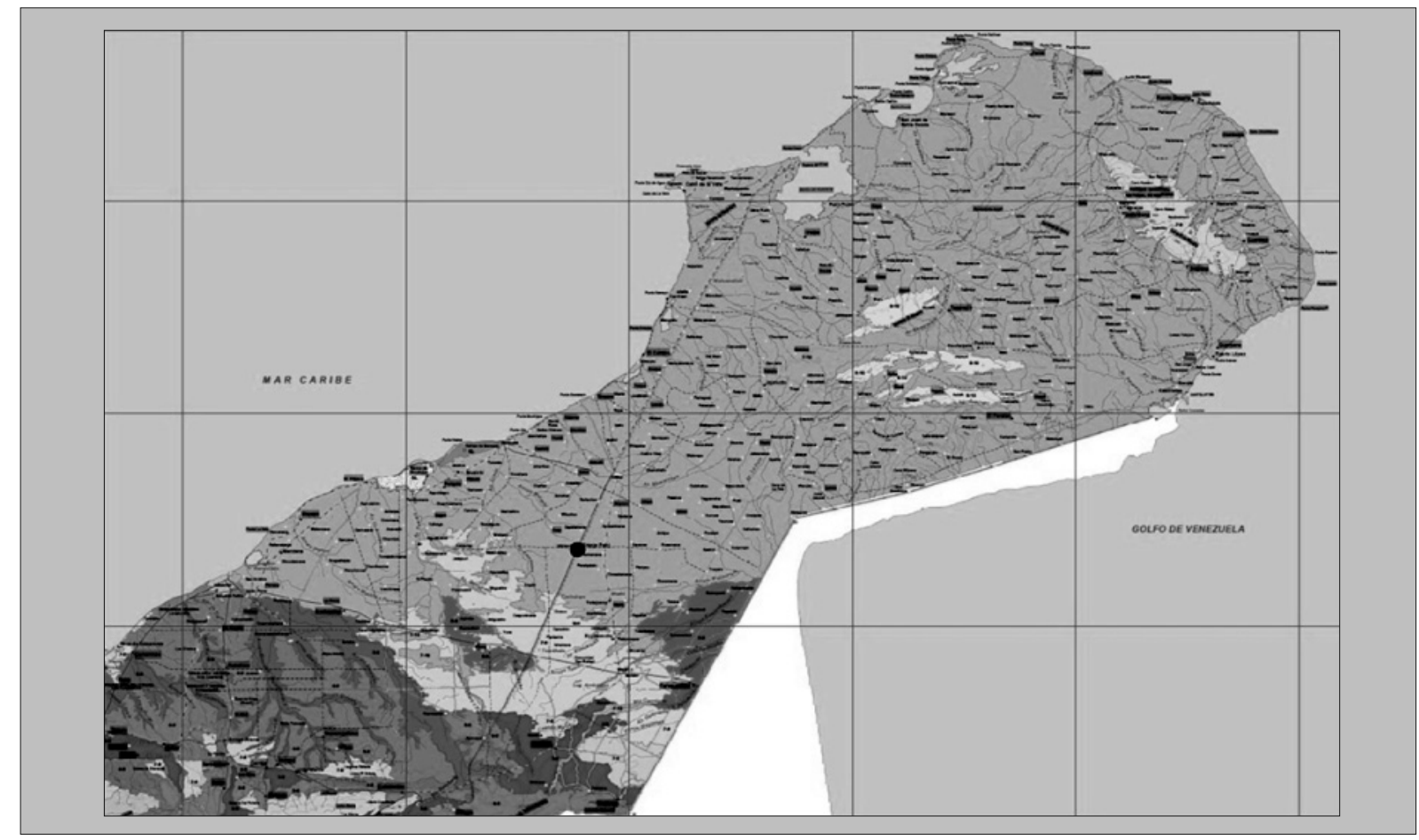

Figura 1. Ubicación del proyecto en el punto señalado

Fuente: tomada de [11].

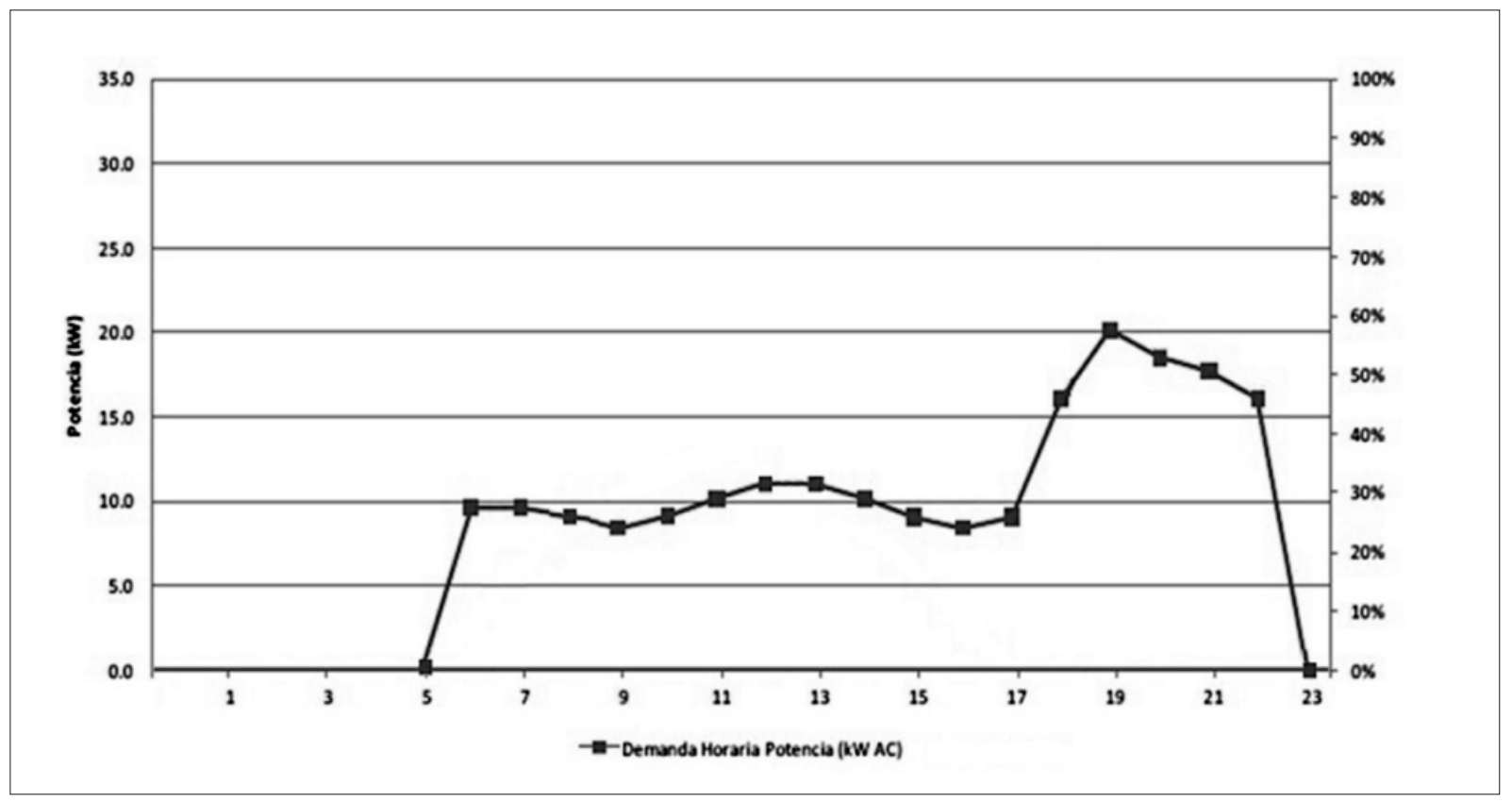

Figura 2. Escenario de demanda D11

Fuente: tomada de [14]. 
Paici, fueron comprados en el Instituto de Hidrología, Meteorología y Estudios Ambientales (Ideam) [13].

Por su parte, para diseñar la demanda de energía requerida por las diferentes comunidades de las ZNI, preferiblemente se emplea la curva de demanda en $\mathrm{kW}$. El escenario de demanda utilizado fue D11, el cual según el Consorcio Energético Corpoema [14] corresponde a la implementación de un sistema híbrido utilizando energía solar, generación con diésel y uso racional de la energía. El escenario demanda energía durante 17 horas (figura 2), suministrando la energía necesaria a 49 usuarios, de los cuales 4 son usuarios oficiales (2 colegios, puesto de salud y comedor comunitario) y 3 son usuarios de pequeños negocios.

\subsection{Evaluación técnica}

En el presente literal se describen técnicamente los componentes elegidos para modelar el HPWS teniendo en cuenta las mejores ofertas del mercado. En primer lugar, se supondrá que Colombia posee completa ausencia de desarrollo industrial en este sector, por lo cual es necesario importar los equipos de generación y control necesarios del sistema. Sin embargo, el número de equipos que se pueden traer depende del número de equipos que caben en los diferentes contenedores que son transportados por buques marítimos. Para ilustrar, existen tres tipos de contenedores, ellos son: de $33,2 \mathrm{~m}^{3}$, de $67,6 \mathrm{~m}^{3} \mathrm{y}$ de $76,2 \mathrm{~m}^{3}$.

\subsubsection{Descripción técnica de los componentes del Hybrid Photovoltaic Wind System (HPWS)}

Para el arreglo fotovoltaico se utilizaron paneles solares monocristalinos de $100 \mathrm{Wp}$ (potencia pico - Wp) prototipo ZX-M, cotizados en la compañía A. Cada panel tiene un área de $0,636 \mathrm{~m}^{2}$ y se caracteriza por presentar un potencial a circuito abierto de 21,6 V con corriente de corto circuito de 4,63 A.

Por su parte, para el arreglo del sistema eólico se utilizaron turbinas eólicas de $5000 \mathrm{Wp}$ prototipo E, cotizadas en la compañía B, cada una de las cuales tiene un rotor de 6,4 metros de diámetro y se caracteriza por presentar una velocidad de arranque de $2,5 \mathrm{~m} / \mathrm{s}$ y una velocidad nominal de $10 \mathrm{~m} / \mathrm{s}$.

Entre tanto, para el arreglo del banco de baterías se utilizaron 12 celdas de $2 \mathrm{~V} / 1000$ Ah prototipo REX-1000 conectadas en serie para un total de $24 \mathrm{~V}$ la unidad. Estas baterías fueron cotizadas en la compañía $C$.

Por último y para describir el sistema de apoyo, es importante mencionar que los inversores de corriente son los principales componentes que conforman el sistema soporte del HPWS. Para ilustrar, se dispuso que el corazón del arreglo fotovoltaico esté compuesto por un inversor de la compañía D (Sunny Mini Central 8000 TL) que transforma la corriente continua en corriente alterna, la cual es despachada directamente a la red de distribución sin necesidad de transformador. Paralelamente a ello, el componente más importante del sistema eólico está conformado por un inversor de la compañía D (WindyBoy 5000 TL) que análogamente, despacha la corriente alterna a la red de distribución. No obstante, para asegurar el abastecimiento de las redes eléctricas en regiones aisladas (off-grid), la compañía desarrolló el ondulador electrónico de baterías Sunny Island 5048, el cual se encarga de crear una red de corriente aislada estable mientras mantiene la tensión y la frecuencia de la red de distribución. En caso de excedente de energía, el Sunny Island carga las baterías; y en caso contrario, suministra la corriente del banco de baterías a la red. Cabe agregar 3 cosas: la primera, es necesario adicionar un gabinete (Multiclúster) para incorporar y acomodar hasta 6 Sunny Island; la segunda, el 
sistema completo está diseñado para contener 3 redes de distribución de baja tensión a $240 \mathrm{~V}$; y la tercera, no podemos ignorar algunos elementos clave, entre ellos el cableado, las estructuras metálicas, el concreto, las canalizaciones, las puestas a tierra, los sistemas de protección, entre otros.

\subsection{Evaluación económica}

Antes que nada, es importante mencionar que la evaluación económica fue realizada para un periodo proyectado de 25 años a una tasa de descuento del 14,7\% anual. Esta tasa fue calculada el 22 de abril de 2009 en el vigésimo documento CREG [15] (Res. 27 de 2009) usando la metodología del Costo Promedio Ponderado de Capital (Weighted Average Cost of Capital [WACC]), la cual es la tasa más extendida y utilizada para descontar los flujos de caja futuros a la hora de valorar un proyecto de inversión. Cabe resaltar que el WACC no es ni un costo ni una rentabilidad exigida, sino un promedio ponderado entre ambas [16]. De igual forma, cabe señalar que el valor de la inflación utilizado en la evaluación fue del $3,17 \%$, valor que fue alcanzado en 2010 .

\subsubsection{Descripción económica de los componentes del Hybrid Photovoltaic Wind System (HPWS)}

Los paneles solares caracterizados en la sección anterior fueron cotizados a un costo de 119 USD la unidad en el Puerto de Carga de Shanghái (Free OnBoard [FOB]). Para el transporte de los paneles solares hasta Colombia, se cotizó un contenedor de $33,2 \mathrm{~m}^{3}$ con 585 paneles solares abordo. El transporte de cada unidad fue avaluado en 5 USD. Entre tanto, el costo del servicio que presta el operador logístico internacional fue cotizado en 18,6 USD por panel, así como el arancel de entrada fue ubicado en $0 \%$ (posición $8541.40 .10 .00)$ sin exención de IVA. Adicionalmente, es importante anotar que los costos de las obras civiles, ingeniería, instalaciones e imprevistos fueron estimados en un solo valor equivalente al $25 \%$ del costo de cada panel, IVA incluido.

Con esta información, es posible calcular el valor del costo de instalación en USD/kW antes de mantenimiento y desmantelamiento (2067 USD/ $\mathrm{kW})$. No obstante, para calcular estos 2 últimos valores, se realizó una pequeña evaluación económica en la cual los costos de mantenimiento fueron estimados en su totalidad en 13,6 USD por panel utilizando la ecuación $8, y$, partiendo del hecho de que en el primer año el costo del mantenimiento fue del $1 \%$ del costo inicial.

$$
C_{\text {amain }}(n)=C_{\text {amain }}(1) \cdot(1+f)^{n}
$$

Finalmente, para el costo del desmantelamiento se utilizó la metodología de los costos nivelados de la International Energy Agency (IEA), en la cual se aclara que dichos costos deben de estimarse con el $5 \%$ de los costos de construcción, cuyo porcentaje se estima alrededor del $13,5 \%$, para un total de 1,4 USD. La estructura global de costos se relaciona en la tabla 1 .

Entre tanto, las turbinas eólicas caracterizadas en la sección anterior, fueron cotizadas a un costo de 4200 USD la unidad FOB Shanghái. Para el transporte de las turbinas eólicas hasta Colombia, se cotizó el transporte de cada una de ellas en 500 USD. El resto de los costos hasta IVA (posición 8412.80.10.00), se estimaron en las mismas proporciones planteadas en el arreglo fotovoltaico.

Para calcular los costos de mantenimiento se utilizó un costo del $3 \%$ para el primer año, dando como resultado un costo presente de 1547,6 USD del mantenimiento de por vida por cada turbina. Entre tanto, para los costos de desmantelamiento, estos fueron calculados de la misma manera que fueron calculados los del sistema fotovoltaico, 
Tabla 1. Estructura de costos del panel solar de 100 Wp

\begin{tabular}{|l|c|}
\hline \multicolumn{1}{|c|}{ COTIZACIÓN PV/100W } & COSTOS (USD) \\
\hline Franco a Bordo (OB) en Shanghái & 119,0 \\
\hline Transporte & 5,0 \\
\hline Operador & 18,6 \\
\hline Arancel & 0,0 \\
\hline Impuesto al Valor Agregado & 22,8 \\
\hline Otros & 41,3 \\
\hline TOTAL & 206,7 \\
\hline Operación y Mantenimiento & 13,6 \\
\hline Desmantelamiento & 1,4 \\
\hline
\end{tabular}

Fuente: elaboración propia.

Tabla 2. Estructura de costos de la turbina eólica de $5000 \mathrm{Wp}$

\begin{tabular}{|l|c|}
\hline \multicolumn{1}{|c|}{ COTIZACIÓN WT/5KW } & COSTOS (USD) \\
\hline Franco a Bordo en Shanghái & 4200,0 \\
\hline Transporte & 500,00 \\
\hline Operador & 705,0 \\
\hline Arancel & 0,0 \\
\hline Impuesto al Valor Agregado & 864,8 \\
\hline Otros & 1567,4 \\
\hline TOTAL & 7837,3 \\
\hline Operación y Mantenimiento & 1547,6 \\
\hline Desmantelamiento & 52,9 \\
\hline
\end{tabular}

Fuente: elaboración propia..

Tabla 3. Estructura de costos de la torre de $16 \mathrm{~m}$ que sostiene cada turbina eólica

\begin{tabular}{|l|c|}
\hline \multicolumn{1}{|c|}{ COTIZACIÓN TORRE/16mt } & COSTOS (USD) \\
\hline Franco a Bordo (OB) en Shanghái & 4880,0 \\
\hline Transporte & 1456,0 \\
\hline Operador & 950,4 \\
\hline Arancel & 728,6 \\
\hline Impuesto al Valor Agregado & 1282,4 \\
\hline Otros & 2324,4 \\
\hline TOTAL & 11621,8 \\
\hline Operación y Mantenimiento & 1988,9 \\
\hline Desmantelamiento & 78,4 \\
\hline
\end{tabular}

Fuente: elaboración propia. dando como resultado 52,9 USD como costo presente. La estructura global de costos se relaciona en la tabla 2.

Cabe señalar que los costos de la torre que sostiene cada turbina fueron cotizados de manera independiente a un costo de 4880 USD la unidad FOB Shanghái. Para el transporte, se cotizó un 1456 USD. Por su parte, los demás costos fueron calculados utilizando las mismas proporciones usadas por Hongxing et ál. [2]. La estructura global de costos se relaciona en la tabla 3.

Por otro lado, las baterías caracterizadas en la sección anterior fueron cotizadas a un costo de 260 USD la unidad FOB Shanghái. Para el transporte de las baterías hasta Colombia, se cotizó una paleta de carga ( 24 paletas por contenedor) con capacidad para 24 baterías, al final el transporte de cada unidad fue evaluado en 11,2 USD. El resto de los costos, incluyendo el IVA, fueron calculados usando las mismas proporciones planteadas en el arreglo fotovoltaico. La estructura de costos se relaciona en la tabla 4 .

Por cierto, los costos de los inversores señalados anteriormente fueron cotizados en la compañía E como sigue: el inversor Sunny Mini Central $8000 \mathrm{TL}$ a un precio de 5470 USD la unidad,

Tabla 4. Estructura de costos de la batería de 1000 $\mathrm{Ah} / 2 \mathrm{~V}$

\begin{tabular}{|l|c|}
\hline \multicolumn{1}{|c|}{ COTIZACIÓN REX/1000 } & COSTOS (USD) \\
\hline Franco a Bordo (OB) en Shanghái & 260,0 \\
\hline Transporte & 11,2 \\
\hline Operador & 40,7 \\
\hline Arancel & 15,6 \\
\hline Impuesto al Valor Agregado & 52,4 \\
\hline Otros & 95,0 \\
\hline TOTAL & 474,8 \\
\hline Operación y Mantenimiento & 103,1 \\
\hline Desmantelamiento & 3,2 \\
\hline
\end{tabular}

Fuente: elaboración propia. 
Tabla 5. Estructura de costos de la grúa hidráulica

\begin{tabular}{|l|c|}
\hline \multicolumn{1}{|c|}{ COTIZACIÓN Grúa/ 2 Cilindros } & COSTOS (USD) \\
\hline Franco a Bordo en Shanghái & 2800,0 \\
\hline Transporte & 500,0 \\
\hline Operador & 495,0 \\
\hline Arancel & 0,0 \\
\hline Impuesto al Valor Agregado & 607,2 \\
\hline Otros & 1100,5 \\
\hline TOTAL & $\mathbf{5 5 0 2 , 8}$ \\
\hline Operación y Mantenimiento & 181,1 \\
\hline Desmantelamiento & 37,1 \\
\hline
\end{tabular}

Fuente: elaboración propia.

el inversor Sunny Island 5048 a un precio de 6450 USD la unidad, el inversor WindyBoy 5000TL aun precio de 4320 USD la unidad, y, el gabinete para incorporar y proteger hasta 6 Sunny Island a un precio de 6120 USD. Cabe agregar que el costo de los elementos de cableado, estructuras metálicas, puestas a tierra, sistemas de protección y pequeñas torres de distribución, fue estimado en un valor global equivalente al $10 \%$ del costo total de todos los aparatos que se necesitan acometer. De aquí se deriva que la red de distribución puede ser modelada para trabajar de manera trifásica y a baja tensión $(240 \mathrm{~V})$.

Para finalizar, falta agregar la grúa hidráulica responsable de elevar las turbinas eólicas. Sus costos pueden ser apreciados en la tabla 5 .

\subsection{Simulación del HPWS en ZNI}

La minimización de las funciones LPSP y ACS se llevó a cabo empleando el programa desarrollado en Matlab, el cual tiene en cuenta los costos de inversión (Equipos, Obras Civiles, Ingeniería, Instalación, Imprevistos, Transporte, Impuestos y otros), los costos de reemplazo de las baterías, los costos de mantenimiento y los costos de desmantelamiento, acorde con la metodología de los costos anuales de Hongxing et ál. [2], y la de costos nivelados de generación [6] - [8].

Adicionalmente, para poder realizar la optimización es importante conocer el espacio de búsqueda, el cual está limitado por el manejo de materiales y de productos de importación. Para ello, se ha cotizado previamente un contenedor de $33,2 \mathrm{~m}^{3}$ de 585 paneles, otro de $33,2 \mathrm{~m}^{3}$ para 3 turbinas de $16 \mathrm{~m}$, y 10 paletas para las baterías. Tras el análisis de la información anterior, los límites de búsqueda finalmente quedan conformados de la siguiente manera: el inferior, compuesto por 1 panel, 0 turbinas y 1 banco de baterías ([1 $\left.\left.\begin{array}{ll}1 & 1\end{array}\right]\right)$, y el superior, conformado por 585 paneles, 3 turbinas y 20 bancos de baterías

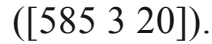

Entre tanto, la figura 3 esboza el gráfico resultante de la simulación del sistema híbrido con los componentes descritos en las secciones previas.

En la figura 3 podemos observar un comportamiento inversamente proporcional entre las 2 variables objetivo evaluadas (LPSP y ACS). Sin embargo, es interesante analizar que para los LPSP menores de $10 \%$ se observa un crecimiento mucho más notable al resto de puntos (Aumento de ACS). Por esta razón, es evidente que la solución que mejor combina los objetivos evaluados es la número 30 de la tabla 6 , la cual se encuentra dentro de las 37 configuraciones que forman parte del conjunto con un LPSP menor a $10 \%$.

Por último, es importante mencionar que la solución número 30 debe pasar por una última restricción, la cual está relacionada con el tamaño de los inversores y su dependencia con la eficiencia de generación de corriente del arreglo fotovoltaico. Para ello, la solución fue paralelamente modelada con el software Sunny Design de inversores de 


\section{investigación}

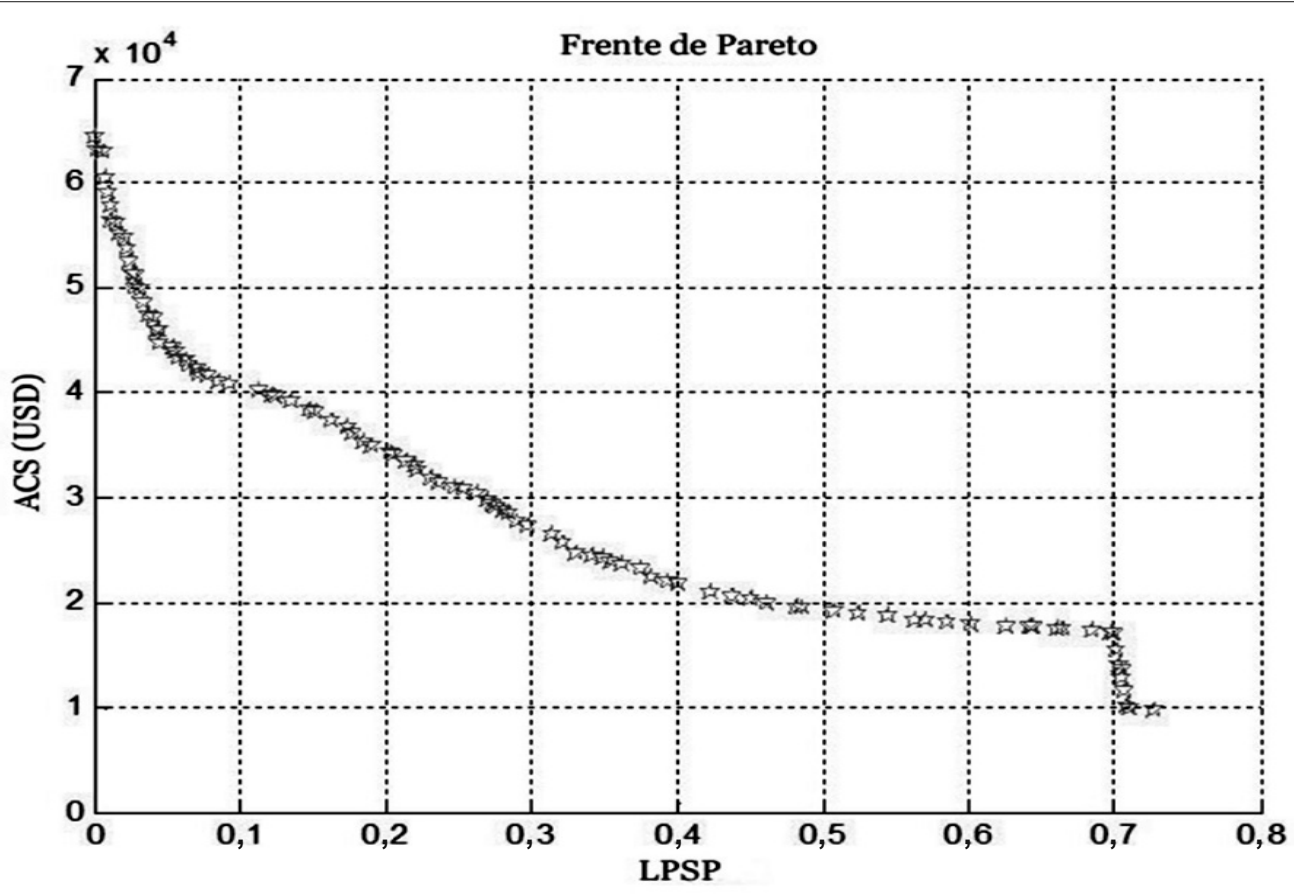

Figura 3. Frente de Pareto

Fuente: elaboración propia.

Tabla 6. Resultados óptimos para el sistema híbrido eólico - solar

\begin{tabular}{|c|c|c|c|c|c|c|c|c|c|}
\hline Config. & Npv & Nwt & Nbat & Hwt & LPSP (\%) & ACS & LCOE & NPC & Egen \\
\hline 1 & 578 & 2 & 16 & 16 & 0,00 & 64,371 & 75,1 & 423,699 & 85,712 \\
\hline 2 & 577 & 2 & 16 & 16 & 0,00 & 64,326 & 75,2 & 423,404 & 85,577 \\
\hline 3 & 579 & 2 & 15 & 16 & 0,28 & 63,094 & 73,5 & 415,293 & 85,848 \\
\hline 4 & 577 & 2 & 15 & 16 & 0,69 & 63,004 & 73,6 & 414,702 & 85,577 \\
\hline 5 & 575 & 2 & 15 & 16 & 0,69 & 62,914 & 73,8 & 414,111 & 85,307 \\
\hline 6 & 574 & 2 & 15 & 16 & 0,69 & 62,870 & 73,8 & 413,815 & 85,171 \\
\hline 7 & 579 & 2 & 13 & 16 & 0,83 & 60,450 & 70,4 & 397,889 & 85,848 \\
\hline 8 & 578 & 2 & 12 & 16 & 0,97 & 59,083 & 68,9 & 388,891 & 85,712 \\
\hline 9 & 578 & 2 & 11 & 16 & 1,11 & 57,761 & 67,4 & 380,189 & 85,712 \\
\hline 10 & 578 & 2 & 10 & 16 & 1,25 & 56,439 & 65,8 & 371,487 & 85,712 \\
\hline 11 & 572 & 2 & 10 & 16 & 1,53 & 56,169 & 66,2 & 369,714 & 84,901 \\
\hline
\end{tabular}




\section{investigación}

\begin{tabular}{|c|c|c|c|c|c|c|c|c|c|}
\hline Config. & Npv & Nwt & Nbat & Hwt & LPSP (\%) & ACS & LCOE & NPC & Egen \\
\hline 12 & 572 & 2 & 9 & 16 & 2,08 & 54,847 & 64,6 & 361,011 & 84,901 \\
\hline 13 & 578 & 1 & 11 & 16 & 2,22 & 53,780 & 65,6 & 353,986 & 81,952 \\
\hline 14 & 577 & 1 & 11 & 16 & 2,36 & 53,735 & 65,7 & 353,690 & 81,817 \\
\hline 15 & 579 & 1 & 10 & 16 & 2,50 & 52,503 & 64,0 & 345,579 & 82,087 \\
\hline 16 & 576 & 0 & 13 & 0 & 2,64 & 51,334 & 65,9 & 337,889 & 77,921 \\
\hline 17 & 573 & 0 & 13 & 0 & 2,78 & 51,200 & 66,1 & 337,003 & 77,515 \\
\hline 18 & 577 & 0 & 12 & 0 & 2,92 & 50,057 & 64,1 & 329,483 & 78,056 \\
\hline 19 & 573 & 0 & 12 & 0 & 3,19 & 49,878 & 64,3 & 328,301 & 77,515 \\
\hline 20 & 575 & 0 & 11 & 0 & 3,33 & 48,645 & 62,5 & 320,189 & 77,786 \\
\hline 21 & 574 & 0 & 11 & 0 & 3,47 & 48,600 & 62,6 & 319,894 & 77,650 \\
\hline 22 & 578 & 0 & 10 & 0 & 3,75 & 47,458 & 60,7 & 312,374 & 78,191 \\
\hline 23 & 573 & 0 & 10 & 0 & 4,17 & 47,233 & 60,9 & 310,896 & 77,515 \\
\hline 24 & 576 & 0 & 9 & 0 & 4,31 & 46,046 & 59,1 & 303,081 & 77,921 \\
\hline 25 & 575 & 0 & 9 & 0 & 4,44 & 46,001 & 59,1 & 302,785 & 77,786 \\
\hline 26 & 577 & 0 & 8 & 0 & 4,58 & 44,769 & 57,4 & 294,674 & 78,056 \\
\hline 27 & 569 & 0 & 8 & 0 & 5,42 & 44,410 & 57,7 & 292,310 & 76,974 \\
\hline 28 & 565 & 0 & 8 & 0 & 5,42 & 44,230 & 57,9 & 291,128 & 76,433 \\
\hline 29 & 560 & 0 & 8 & 0 & 5,69 & 44,006 & 58,1 & 289,650 & 75,757 \\
\hline 30 & 574 & 0 & 7 & 0 & 5,83 & 43,312 & 55,8 & 285,085 & 77,650 \\
\hline 31 & 569 & 0 & 7 & 0 & 6,39 & 43,088 & 56,0 & 283,608 & 76,974 \\
\hline 32 & 562 & 0 & 7 & 0 & 6,53 & 42,773 & 56,3 & 281,539 & 76,027 \\
\hline 33 & 551 & 0 & 7 & 0 & 7,08 & 42,279 & 56,7 & 278,289 & 74,539 \\
\hline 34 & 542 & 0 & 7 & 0 & 7,36 & 41,875 & 57,1 & 275,629 & 73,322 \\
\hline 35 & 537 & 0 & 7 & 0 & 7,92 & 41,651 & 57,3 & 274,151 & 72,645 \\
\hline 36 & 525 & 0 & 7 & 0 & 8,61 & 41,112 & 57,9 & 270,605 & 71,022 \\
\hline 37 & 517 & 0 & 7 & 0 & 9,31 & 40,753 & 58,3 & 268,241 & 69,940 \\
\hline 38 & 522 & 0 & 7 & 0 & 9,03 & 41,112 & 58,2 & 270,605 & 70,616 \\
\hline
\end{tabular}

Fuente: elaboración propia. 


\section{investigación}

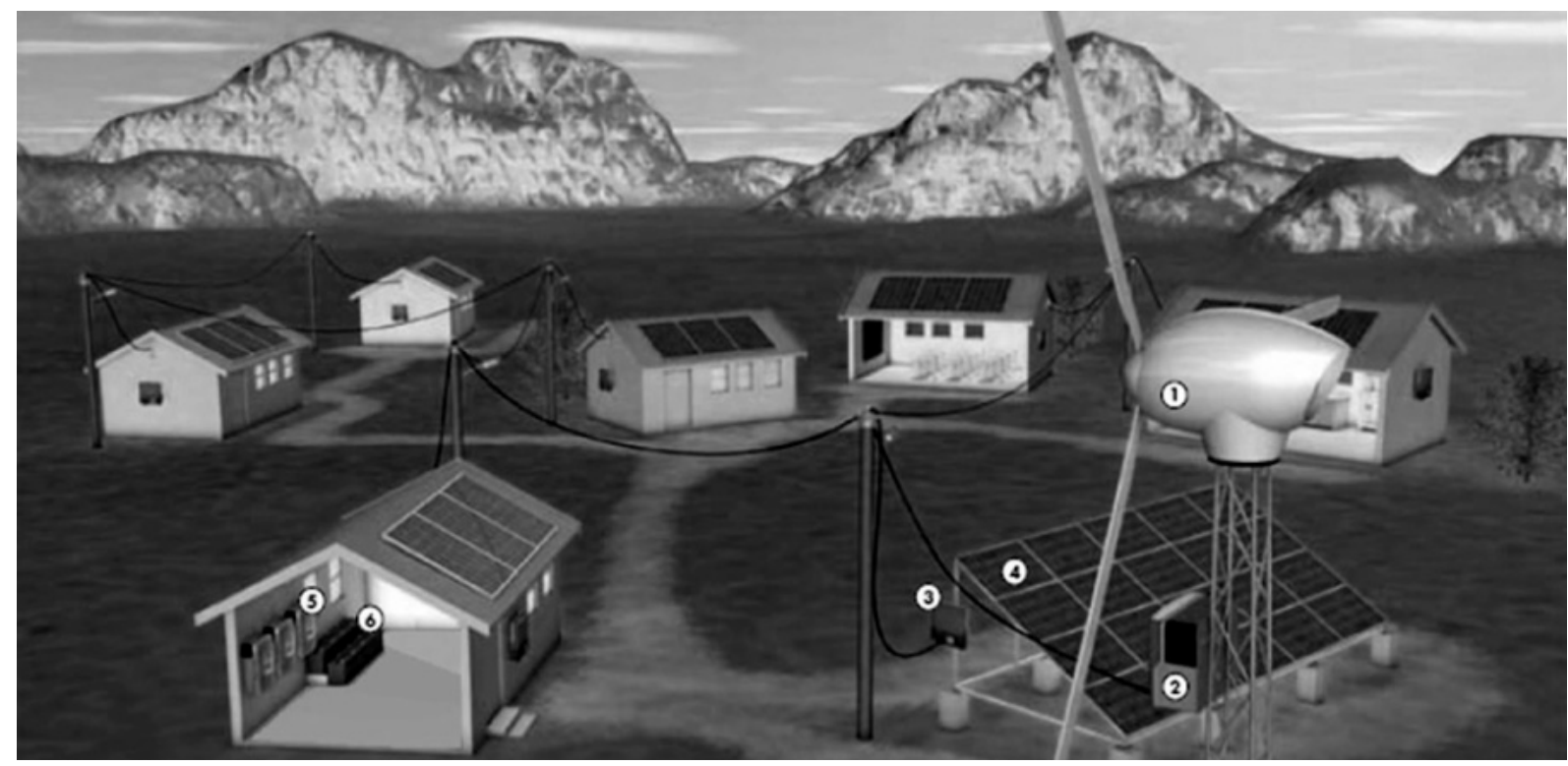

Figura 4. Modelado del sistema con los componentes necesarios para su correcto funcionamiento

Nota: 1) turbina eólica, 2) inversor Windyboy 5000 TL, 3) inversor Sunny Mini Central 8000 TL, 4) arreglo fotovoltaico, 5) inversores Sunnylsland, 6) banco de baterías

Fuente: Tomada y modificada de Solar Technology [17].

la compañía D [17], a fin de obtener la configuración óptima que asegure la compatibilidad y el mejor compromiso entre los inversores y el $N_{p v^{*}}$ Como resultado final, la configuración fue restringida a 522 paneles solares a fin de establecer una inyección trifásica eficiente usando 6 inversores de tipo 8000TL.

Con todo y lo anterior, la solución fue nuevamente determinada y la nueva solución ajustable fue la número 36 , que más tarde fue reestructurada y renombrada en la casilla número 38 debido al exceso de 3 paneles solares que al final quedan disponibles para futuros imprevistos.

\subsection{Discusión}

El método técnico económico propuesto fue aplicado para diseñar y modelar un HPWS que permitiese suministrar la energía requerida del escenario D11, en la Granja Paicí, utilizando los componentes anteriormente descritos (figura 4). En primera instancia, es indudable que las configuraciones 1 y 2 son las más confiables a pesar de que resulten ser las más onerosas. Aquí, teóricamente no existe riesgo alguno de perder el suministro de energía. Además es evidente que las primeras 15 configuraciones presentan complementariedad. Por otro lado, en la figura 3 es claro que existe un comportamiento inversamente proporcional entre los 2 objetivos evaluados (LPSP y ACS), y como podemos observar, este comportamiento es mucho más marcado después de superada una confiabilidad del $90 \%$ (LPSP $<10 \%$ ). Por esta razón, las soluciones más cercanas a este tipo de valores resultan ser las más interesantes.

De acuerdo con el literal anterior, la solución 38 es la que mejor representa una combinación en términos de confiabilidad y costo. Además, si miramos desde una perspectiva netamente económica podemos notar que aquella sugiere utilizar solo 
un sistema fotovoltaico en vez de un sistema híbrido. En efecto, en estas circunstancias se considera que el sistema híbrido no es complementario probablemente debido a 2 factores: el primero, las torres son demasiado costosas, y el segundo, la altura de 16 metros no es eficiente para aprovechar una cinética de vientos apropiada en el escenario evaluado. De aquí se deriva que sería factible explorar e incentivar empresas colombianas que fabriquen las torres de las turbinas eólicas que entren al país, y segundo, evaluar la posibilidad de realizar la simulación utilizando otro tipo de turbinas eólicas que puedan ubicarse a una altura superior. De esta forma se estima que la complementariedad del HPWS podría llegar a ser más fácilmente visualizada (en muchas más configuraciones) en trabajos de modelación posteriores, incluso con mejores resultados económicos que el costo nivelado de generación de la configuración 38 , la cual reporta una confiabilidad del $91 \%$ para el mes con menor recurso y un costo nivelado de $58,2 \mathrm{cUSD} / \mathrm{kWh}$.

Como ya se mencionó, los valores utilizados en la modelación del sistema fueron los del mes con menor recurso. Esto debido a varias razones entre las cuales se encuentran: 1) incapacidad económica para adquirir un histórico de datos suficiente (para modelar una distribución de probabilidad) y 2) escasa disponibilidad de estaciones especializadas que suministren este tipo de información (radiación y velocidad de viento horaria). En ese sentido, en esta investigación se considera que multiplicar por 12 (meses) la máxima energía eléctrica que es capaz de entregar la planta de generación en condiciones extremas de bajo recurso, no deja de ser una aproximación empírica viable y económica para calcular prudentemente la energía firme de un sistema híbrido cuando básicamente no existe información histórica meteorológica.

Con todo y lo anterior se estima que, usando la configuración 38 lo más seguro es que lleguen a existir muy buenos excedentes que tienen el potencial de ser vendidos en una microred de generación distribuida local. En consecuencia, para la correcta interpretación de resultados podemos hacer uso de lo indicado en el literal 6.1 del volumen III del PDFNCE [4], en el cual se establece un factor de corrección para la energía solar (tabla 7) con el ánimo de escalar regionalmente los costos de generación cuando sea necesario.

De esta forma, si la configuración 38 efectivamente se gestiona usando el promedio anual del mes con menor recurso (octubre de 2008 - 4,6 $\mathrm{kWh} / \mathrm{m}^{2}$ ), el factor multiplicador para escalar el promedio usado al promedio real del sitio sería de 1,17 veces (promedio de radiación anual local [5,4 kWh/ $\left.\left./ \mathrm{m}^{2} / \mathrm{año}\right]\right)$. Por esta razón, en realidad se presentarían 3 situaciones teóricamente factibles: la primera, que la energía realmente generada en el año sería 1,17 veces mayor a la especificada en la tabla 6; la segunda, que el costo nivelado real-

Tabla 7. Factor de corrección para energía solar

\begin{tabular}{|c|c|}
\hline $\begin{array}{c}\text { Radiación solar } \\
\text { (kWh/m2/día) }\end{array}$ & $\begin{array}{c}\text { Factor corrección } \\
\text { del FC }\end{array}$ \\
\hline $6 \cdot 4$ & $\mathbf{1 , 3 3}$ \\
\hline 6,2 & $\mathbf{1 , 2 9}$ \\
\hline 6,0 & $\mathbf{1 , 2 5}$ \\
\hline 5,8 & $\mathbf{1 , 2 1}$ \\
\hline 5,6 & $\mathbf{1 , 1 7}$ \\
\hline 5,4 & $\mathbf{1 , 1 3}$ \\
\hline 5,2 & $\mathbf{1 , 0 8}$ \\
\hline 5,0 & $\mathbf{1 , 0 4}$ \\
\hline 4,8 & $\mathbf{1 , 0 0}$ \\
\hline 4,6 & $\mathbf{0 , 9 6}$ \\
\hline 4,4 & $\mathbf{0 , 9 2}$ \\
\hline 4,2 & $\mathbf{0 , 8 8}$ \\
\hline 4,0 & $\mathbf{0 , 8 3}$ \\
\hline 3,8 & $\mathbf{0 , 7 9}$ \\
\hline 3,6 & $\mathbf{0 , 7 5}$ \\
\hline Caso base $=4,8 \mathrm{kWh} / \mathrm{m}^{2} / \mathrm{día}$ \\
\hline
\end{tabular}

Fuente: tomada de [14] 


\section{investigación}

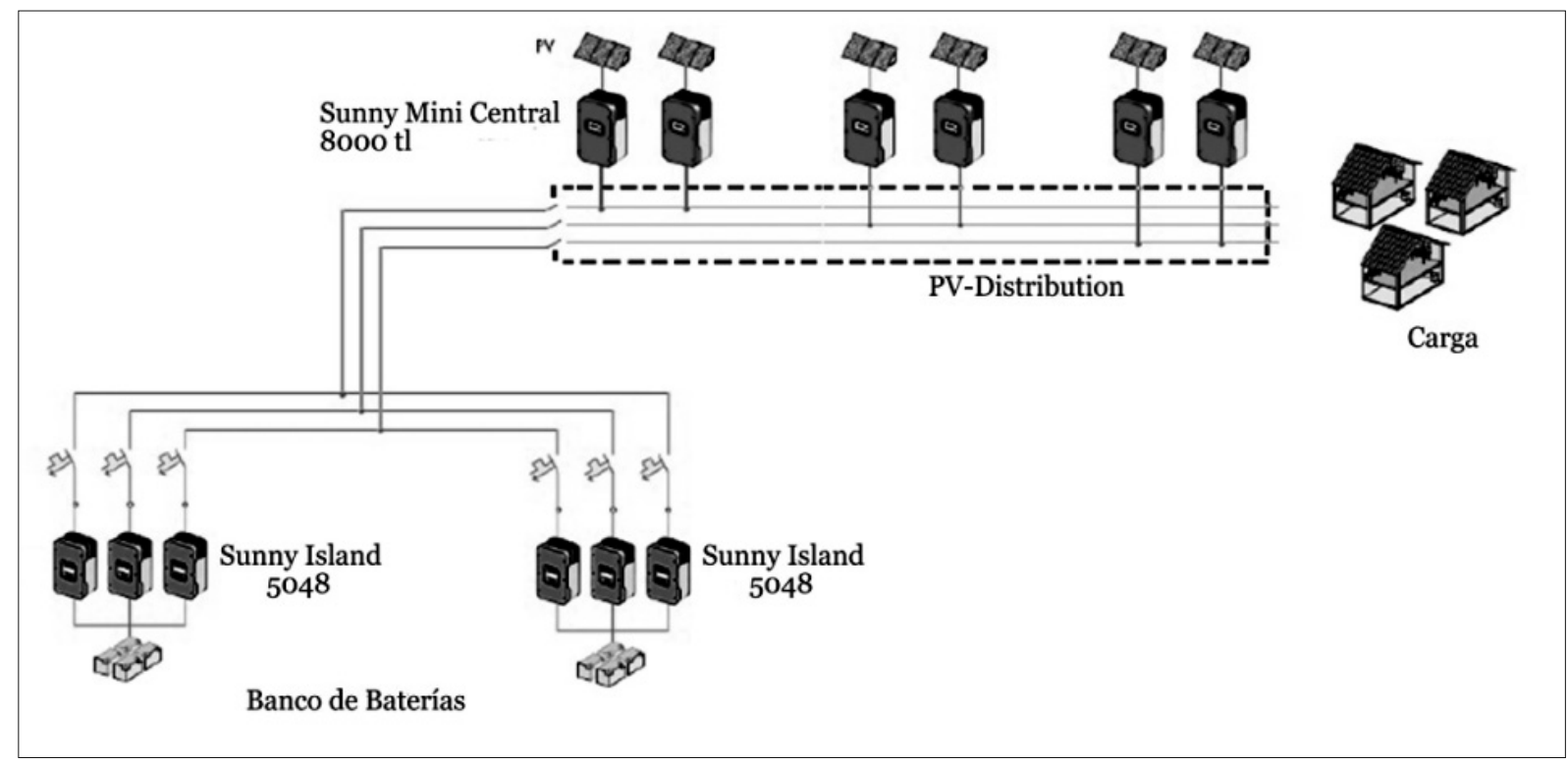

Figura 5. Sistema a implementar

Fuente: tomada de [14].

mente generado sería de 49,76 cUSD/kWh $(58,2$ $\mathrm{cUSD} / \mathrm{kWh} / 1,17)$, revelando así que el costo de los sistemas ha empezado a ser competitivo con el diésel (0,44-1,5 USD/kWh) de las ZNI [14], a pesar de que el costo de las baterías detente más del $50 \%$ del total de los costos; y la tercera, que la confiabilidad real sería mucho mayor que el $91 \%$ declarado en la configuración. Para finalizar, es importante reportar que la configuración más óptima desde el punto de vista económico y confiable puede ser observada en la figura 5 .

\section{CONCLUSIONES}

En esta investigación se presentó una metodología para evaluar y diseñar técnica y económicamente sistemas híbridos eólico solares (HPWS por sus siglas en inglés) en Zonas No Interconectadas (ZNI), usando tecnologías de energías renovables (RET, por sus siglas en inglés). La metodología fue utilizada para simular un caso de estudio usando el algoritmo genético multiobjetivo del toolbox de Matlab.
El modelo en general puede ser usado para calcular la configuración óptima de un sistema minimizando la Probabilidad de Pérdida de Suministro Energético (LPSP, por sus siglas en inglés) y los Costos Anualizados (ACS, por sus siglas en inglés). En la simulación, fue hallado un tamaño de diseño óptimo de un HPWS con baterías de almacenamiento, esto para suministrar eficientemente la energía a 49 usuarios de la localidad de la Granja Paicí en Uribia, Guajira. Los resultados obtenidos muestran que existe un comportamiento inversamente proporcional entre los 2 objetivos evaluados (LPSP y ACS), el cual es mucho más pronunciado después de superada una confiabilidad del $90 \%$ (LPSP $<10 \%$ ).

Como resultados principales se encuentra que el costo nivelado de generación (Levelized Cost Of Energy - LCOE) asciende a 49,8 cUSD/kWh $(52,2 \mathrm{~kW})$, de los cuales más del $50 \%$ proviene de las baterías y otros componentes. Lo anterior revela que el costo de este tipo de sistemas ha empezado a cobrar relevancia porque es competitivo 


\section{investigación}

frente a otros tipos de generación como el diesel de las ZNI (0,44-1,5 USD/kWh).

A pesar de la intención por modelar el HPWS, las configuraciones óptimas reportaron que la tecnología fotovoltaica predomina unilateralmente sobre la tecnología eólica, demostrando que el sistema eólico no era complementario económicamente con el fotovoltaico, principalmente porque la altura de trabajo de las turbinas resultó ser insuficiente para extraer la máxima eficiencia cinética del viento (Ineficiencia técnico-económica reflejada en el costo por capacidad - USD/kW). Adicionalmente, otra de las limitaciones que se presentaron en la simulación tiene que ver con el modelamiento técnico, ya que este fue realizado en base a diferentes parámetros teóricos que pueden distar un poco de la realidad. Con todo y lo anterior, se concluye que es fundamental desarrollar proyectos demostrativos dentro de las universidades para poder interpretar y simular en detalle la generación intermitente que este tipo de tecnologías presenta.

\section{AGRADECIMIENTOS}

Los autores agradecen a la Universidad de Antioquia por el apoyo financiero recibido a través del proyecto "sostenibilidad 2013-2014".

\section{REFERENCIAS}

[1] C. Zapata, "Fuentes alternativas de generación de energía, incentivos y mandatos regulatorios: Una aproximación teórica al caso colombiano", Revista Energética, vol. 34, no. 1, pp. 55-63, Dec. 2005.

[2] Y. Hongxing, Z. Wei, and L. Chengzhi, "Optimal design and techno-economicanalysis of a hybrid solar-wind power generation system", Applied Energy, vol. 86, no. 2, pp. 163-169, Feb. 2009.

[3] G. Tina, S. Gagliano, and S. Raiti, "Hybrid solar/wind power system probabilistic modeling for long-term performance assessment", Solar Energy, vol. 80, no. 5, pp. 578-588, May. 2006.

[4] S. Diaf, M. Belhamel, M. Haddadi, and A. Louche, "Technical and economic assessment of hybrid photovoltaic/wind system with battery storage in Corsica island", Energy Policy, vol. 36, no. 2, pp. 743-754, Feb. 2008.
[5] A. Bin, Y. Hongxing, H. Shen, and L. Xianbo, "Computer aided design for PV/ Wind hybrid system", Renewable Energy, vol. 28, no. 10, pp. 1491-1512, Aug. 2003.

[6] Nuclear Energy Agency, International Energy Agency, and Organisation for Economic Co-operation and Development, Projected cost of generating electricity - update. Paris: Head of Publications Service, 2005.

[7] International Energy Agency, Electricity information - IEA statistics book, Paris: IEA, 2010.

[8] K. Branker, M.J.M. Pathak, and J.M. Pearce, "A review of solar photovoltaic levelized cost of electricity", Renewable and Sustainable Energy Reviews, vol. 15, no. 9, pp. 4470-4482, Dec. 2011.

[9] D.Kalyanmoy,Multi-objectiveoptimization using evolutionary algorithms, Chichester: John Wiley and Sons Ltd., 2010. 


\section{investigación}

[10] D. Kalyanmoy, A. Pratap, S. Agarwal, and T. Meyarivan, "A fast elitis non-dominated sorting genetic algorithm for multiobjective optimization: NSGA-II", IEEE Transactions on Evolutionary Computation, vol. 6, no. 2, pp. 181-197, Dec. 2002.

[11] Sistema de Información Ambiental de Colombia, "Estudio general de suelos y zonificación de tierras del departamento de la guajira", SIAC. [En línea]. Disponible en http://www.siac.gov.co/contenido/contenido.aspx? catID $=562 \& \operatorname{conID}=849$

[12] Departamento Administrativo Nacional de Estadística, "Censo general 2005: perfil Uribia - La Guajira,’DANE. [En línea]. Disponible en http://www.dane.gov.co/files/censo2005/perfiles/guajira/uribia.pdf

[13] Instituto de Hidrología Meteorología y Estudios Ambientales, "Compra de información”, IDEAM. [En línea]. Disponible en http://institucional.ideam.gov.co/jsp/index. jsf
[14] Corporación para la Energía y el Medio Ambiente, "Elementos de política, riesgos ante el cambio climático, complementariedad entre las FNCE y el SIN, y costos indicativos de las FNCE. PDFNCE - Volumen III", CORPOEMA. [En línea]. Disponible en http://www.upme.gov.co/Sigic/ DocumentosF/Vol_3_Tecnologia_Costos_ FNCE.pdf

[15] Comisión de Regulación de Energía y Gas, "Resolución Número 27 del 22 de abril de 2009: Documento anexo 20", CREG, [En línea]. Disponible en http://www.creg.gov.co

[16] P. Fernández, "WACC: Definición, Interpretaciones Equivocadas y Errores", IESE, [En línea]. Disponible en http://www.iese. edu/research/pdfs/DI-0914.pdf

[17] Solar Technology, "Sunny design", SMA, [En línea]. Disponible en http://www.smaiberica.com/es/productos/software/sunnydesign.html 